Article

\title{
Partially Coherent Flat-Topped Beam Generated by an Axicon
}

\author{
Minghui Zhang ${ }^{1}$, Xianlong Liu ${ }^{2}$, Lina Guo ${ }^{3}{ }^{-}$, Lin Liu ${ }^{4, *}$ and Yangjian Cai ${ }^{2,4, *}$ \\ 1 School of Physics and Material Science, Anhui University, Hefei 230039, China; zmh@ahu.edu.cn \\ 2 Shandong Provincial Engineering and Technical Center of Light Manipulations \& Shandong Provincial Key \\ Laboratory of Optics and Photonic Device, School of Physics and Electronics, Shandong Normal University, \\ Jinan 250014, China; xianlongliu@sdnu.edu.cn \\ 3 School of Optoelectronic Engineering, Guangdong Polytechnic Normal University, Guangzhou 510665, \\ China; guoln523@126.com \\ 4 Key Lab of Advanced Optical Manufacturing Technologies of Jiangsu Province \& Key Lab of Modern \\ Optical Technologies of Education Ministry of China, School of Physical Science and Technology, \\ Soochow University, Suzhou 215006, China \\ * Correspondence: liulin@suda.edu.cn (L.L.); yangjiancai@suda.edu.cn (Y.C.); Tel.: +86-1391-263-6612 (L.L.); \\ $+86-531-89611187$ (Y.C.)
}

Received: 23 March 2019; Accepted: 6 April 2019; Published: 11 April 2019

check for updates

\begin{abstract}
The intensity distribution of a partially coherent beam with a nonconventional correlation function, named the multi-Gaussian Schell-model (MGSM) beam, focused by an axicon was investigated in detail. Our numerical results showed that an optical needle with a flat-topped spatial profile and long focal depth was formed and that we can modulate the focal shift and focal depth of the optical needle by varying the width of the degree of coherence (DOC) and the parameters of the correlation function. The adjustable optical needle can be applied for electron acceleration, particle trapping, fiber coupling and percussion drilling.
\end{abstract}

Keywords: coherence and statistical optics; correlation function; axicon; laser beam shaping

\section{Introduction}

Over the past years, axicon lenses have been studied widely due to their unique properties of producing focal lines of narrow transverse width over extended distances [1,2]. The axicon lens and its combination with other optical elements have been applied in many fields such as laser machining, corneal surgery, and atom alignment and trapping [2-5]. Since 1986, when Durnin first put forward a non-diffracting beam named a Bessel beam [6], a great deal of attention has been concentrated on the axicons lens as it provides the most energy efficient method of realizing generally diffraction-free beams [7-9]. Later, the research was extended from completely coherent beams to partially coherent beams. For a spatially partially coherent light, Friberg et al. proposed a method of designing annular-aperture axicons or apodized annular logarithmic axicons to generate a uniform intensity axial line image $[10,11]$. Shukri et al. designed diffractive axicons to produce uniform line images in a twisted Gaussian Schell-model (TGSM) illumination [12,13]. Pu et al. generated adjustable partially coherent bottle beams by focusing partially coherent light with an axicon-lens system [14,15]. Alkelly et al. examined the focal depth of partially coherent fields without aperture [16]. To date, the research on focusing partially coherent fields by an axicon lens has been restricted to Gaussian Schell-model beams. The results of the above-mentioned research show that the coherence length of partially coherent light has an intimate relationship with the axial intensity distribution of the focused field formed by an axicon. In an experiment, we can easily obtain different values of the coherence 
width by varying the focused beam spot on a rotating ground glass disk (RGGD) through adjusting the distance between the focus lens and RGGD [17].

With the rapid development of partially coherent light and its wide applications [17], nonconventional partially coherent light, the correlation function of which does not satisfy a Gaussian distribution, has been proposed $[18,19]$. It has been confirmed both theoretically and experimentally that the correlation function provides a new effective way to shape the intensity profile [20-30]. What is the effect of the nonconventional correlation function on the intensity distribution of beams focused by an axicon lens? Among a variety of nonconventional partially coherent beams, we are more interested in beams with a flat-topped spatial profile, which have shown great value in lithography, integrated circuit trimming, laser welding, and biomedical engineering. In 2012, a multi-Gaussian Schell-model (MGSM) beam [31], the degree of coherence (DOC) of which was modeled by multi-Gaussian distribution, was proposed by Korotkova et al. The MGSM beam can form flat square or rectangular intensity distributions in the far field [31-33]. Later, generalized GMSM beam and elliptical MGSM beam were proposed, which can produce prescribed flat-topped spatial profiles [34,35]. For these nonconventional partially coherent beams, extra parameters of a correlation function can be used to control the flat-topped spatial profiles. In this paper, we will investigate the intensity distribution of a MGSM beam focused by an axicon and explore the effect of a nonconventional correlation function on the focusing properties of a MGSM beam.

\section{Analytical Expression of a MGSM Beam Focused by an Axicon Lens}

The cross-spectral density (CSD) function of a MGSM beam in the source plane is described as follows [31]:

$$
W\left(\boldsymbol{\rho}_{1}, \boldsymbol{\rho}_{2}\right)=G_{0} \exp \left(-\frac{\boldsymbol{\rho}_{1}^{2}+\boldsymbol{\rho}_{2}^{2}}{2 \sigma^{2}}\right) \mu\left(\boldsymbol{\rho}_{1}, \boldsymbol{\rho}_{2}\right),
$$

where $G_{0}$ is a normalized constant, $\boldsymbol{\rho}_{1}, \boldsymbol{\rho}_{2}$ are two arbitrary points in the source plane, $\sigma$ is the beam waist width of the source, $\mu\left(\boldsymbol{\rho}_{1}, \boldsymbol{\rho}_{2}\right)$ is the spectral DOC. The DOC of a MGSM beam is expressed by [31]:

$$
\mu\left(\boldsymbol{\rho}_{1}, \boldsymbol{\rho}_{2}\right)=\frac{1}{C_{0}} \sum_{m=1}^{M} \frac{(-1)^{m-1}}{m}\left(\begin{array}{c}
M \\
m
\end{array}\right) \exp \left[-\frac{\left(\boldsymbol{\rho}_{2}-\boldsymbol{\rho}_{1}\right)^{2}}{2 m \delta^{2}}\right]
$$

where $C_{0}=\sum_{m=1}^{M} \frac{(-1)^{m-1}}{m}\left(\begin{array}{c}M \\ m\end{array}\right)$ is the normalization factor, $M$ is the beam order and $\delta$ is the initial correlation width of the beam. A MGSM beam would reduce to a GSM beam when $M=1$ or a coherent Gauss beam when $\delta=\infty$ and $M=1$. Figure 1 shows the distribution of the DOC of a MGSM beam for different values of the beam order $M$. Generally, it displays a more complicated form represented by a sum of positive and negative exponentials.

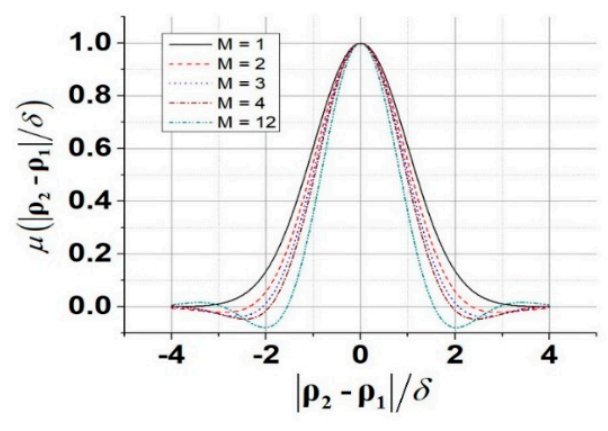

Figure 1. Distribution of the degree of coherence (DOC) of a multi-Gaussian Schell-model (MGSM) beam in the source plane for different values of the beam order $M$. 
According to the generalized Huygens-Fresnel principle [36,37], a MGSM beam propagating through an axicon, as shown on Figure 2, can be dealt with the following generalized Collins integral formula:

$$
\begin{array}{r}
W\left(\mathbf{r}_{1}, \mathbf{r}_{2}\right)=\frac{1}{\lambda^{2} B^{2}} \iint W\left(\rho_{1}, \rho_{2}\right) T^{*}\left(\boldsymbol{\rho}_{1}\right) T\left(\boldsymbol{\rho}_{2}\right) \times \exp \left\{\frac { - j k } { 2 } \left[\left(\frac{A^{*}}{B^{*}} \boldsymbol{\rho}_{1}^{2}-\frac{A}{B} \boldsymbol{\rho}_{2}^{2}\right)\right.\right. \\
\left.\left.-2\left(\frac{1}{B^{*}} \boldsymbol{\rho}_{1} \mathbf{r}_{1}-\frac{1}{B} \boldsymbol{\rho}_{2} \mathbf{r}_{2}\right)+\left(\frac{D^{*}}{B^{*}} \mathbf{r}_{1}^{2}-\frac{D}{B} \mathbf{r}_{2}^{2}\right)\right]\right\} \mathbf{d}^{2} \boldsymbol{\rho}_{1} \mathbf{d}^{2} \boldsymbol{\rho}_{2},
\end{array}
$$

where $\mathbf{r}_{1}, \mathbf{r}_{2}$ are two arbitrary points in the output plane, $\lambda$ is the wavelength, $A, B, C$ and $D$ are the transfer matrix elements of an optical system, $T$ is the transmittance function of the axicon lens, which can be expressed as follows:

$$
T(\rho)= \begin{cases}\exp \left[j k\left(n_{d} \Re-\beta \rho\right)\right] & \rho \leq R \\ 0 & \rho>R^{\prime}\end{cases}
$$

with

$$
\beta=\left(n_{d}-1\right) \tan \frac{\pi-\alpha}{2} .
$$

Here $n_{d}$ is the relative refractive index of the axicon to the medium around it, $\Re$ is the maxima of its thickness, $\alpha$ is the apex angle of the axicon lens.

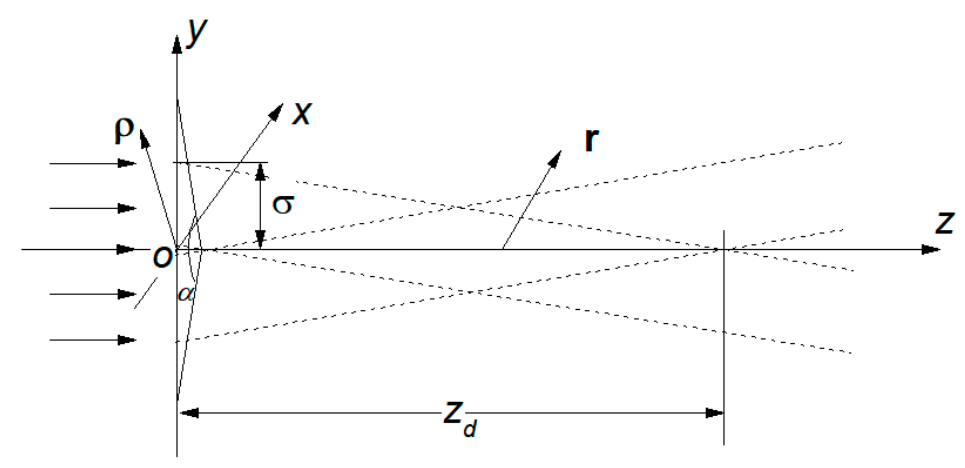

Figure 2. Optical layout of a MGSM beam passing through an axicon lens.

One can rewrite Equation (3) in a polar coordinate system with $\rho=(\rho \cos \varphi, \rho \sin \varphi), \mathbf{r}=$ $(r \cos \theta, r \sin \theta)$, as:

$$
\begin{aligned}
W\left(\mathbf{r}_{1}, \mathbf{r}_{2}\right)= & \frac{1}{\lambda^{2} B^{2}} \frac{1}{C_{0}} \sum_{m=1}^{M}\left(\begin{array}{c}
M \\
m
\end{array}\right) \frac{(-1)^{m-1}}{m} \exp \left[\frac{-j k}{2}\left(\frac{D^{*}}{B^{*}} \mathbf{r}_{1}^{2}-\frac{D}{B} \mathbf{r}_{2}^{2}\right)\right] \\
& \times \int_{0}^{+\infty} \int_{0}^{+\infty} \rho_{1} \rho_{2} \exp \left[-\frac{1}{2 \Delta_{m}^{2}}\left(\rho_{2}^{2}+\rho_{1}^{2}\right)\right] \exp \left[j k f\left(\rho_{1}, \rho_{2}\right)\right] \\
& \times \int_{0}^{2 \pi} \int_{0}^{2 \pi} \exp \left[\frac{\rho_{1} \rho_{2} \cos \left(\varphi_{2}-\varphi_{1}\right)}{m \delta^{2}}\right] \exp \left\{\left[j k \left(\frac{1}{B^{*}} \rho_{1} r_{1} \cos \left(\varphi_{1}-\theta_{1}\right)\right.\right.\right. \\
& \left.\left.\left.-\frac{1}{B} \rho_{2} r_{2} \cos \left(\varphi_{2}-\theta_{2}\right)\right)\right]\right\} d \varphi_{1} d \varphi_{2} d \rho_{1} d \rho_{2}
\end{aligned}
$$

where

$$
\frac{1}{\Delta_{m}^{2}}=\frac{1}{\sigma^{2}}+\frac{1}{m \delta^{2}}
$$

And after tedious integration, the expression of the CSD in the output plane can be obtained as:

$$
f\left(\rho_{1}, \rho_{2}\right)=\left(\frac{A}{2 B} \rho_{2}^{2}-\beta \rho_{2}\right)-\left(\frac{A^{*}}{2 B^{*}} \rho_{1}^{2}-\beta \rho_{1}\right) .
$$




$$
\begin{aligned}
W\left(\mathbf{r}_{1}, \mathbf{r}_{2}\right)= & \frac{k^{2}}{B^{2} C_{0}} \sum_{m=1}^{M} \sum_{l=-\infty}^{\infty} e^{j l\left(\theta_{2}-\theta_{1}\right)}\left(\begin{array}{c}
M \\
m
\end{array}\right) \frac{(-1)^{m-1}}{m} \exp \left[\frac{-j k}{2}\left(\frac{D^{*}}{B^{*}} \mathbf{r}_{1}^{2}-\frac{D}{B} \mathbf{r}_{2}^{2}\right)\right] \\
& \times \int_{0}^{+\infty} \int_{0}^{+\infty} \rho_{1} \rho_{2} \exp \left[-\frac{1}{2 \Delta_{m}^{2}}\left(\rho_{2}^{2}+\boldsymbol{\rho}_{1}^{2}\right)\right] \exp \left[j k f\left(\rho_{1}, \rho_{2}\right)\right] \\
& \times C_{l m}\left(B, \rho_{1}, \rho_{2}, r_{1}, r_{2}\right) d \rho_{1} d \rho_{2}
\end{aligned}
$$

with

$$
C_{l m}\left(B, \rho_{1}, \rho_{2}, r_{1}, r_{2}\right)=j^{-l} J_{l}\left(j \frac{\rho_{1} \rho_{2}}{m \delta^{2}}\right) J_{l}\left(k \frac{\rho_{1} r_{1}}{B^{*}}\right) J_{l}\left(k \frac{\rho_{2} r_{2}}{B}\right) .
$$

where $J_{l}$ is the first kind Bessel function with order $l$. In Equation (9), the phase factor $\exp \left[j k f\left(\rho_{1}, \rho_{2}\right)\right]$ oscillates around zero intensively within the range of the optical frequencies. Applying the stationary phase method in $[12,13,38]$, the integration result of Equation (9) can be acquired:

$$
\begin{aligned}
W\left(\mathbf{r}_{1}, \mathbf{r}_{2}\right)= & \frac{2 \pi k}{B^{2} \sqrt{|a b|} C_{0}} \rho_{1 s} \rho_{2 s} \exp \left[\frac{-j k}{2}\left(\frac{D^{*}}{B^{*}} \mathbf{r}_{1}^{2}-\frac{D}{B} \mathbf{r}_{2}^{2}\right)\right] \exp \left[j k f\left(\rho_{1 s}, \rho_{2 s}\right)\right] \\
& \times \sum_{m=1}^{M} \sum_{l=-\infty}^{\infty}\left(\begin{array}{c}
M \\
m
\end{array}\right) \frac{(-1)^{m-1}}{m} \exp \left[-\frac{1}{2 \Delta_{m}^{2}}\left(\boldsymbol{\rho}_{2 s}^{2}+\boldsymbol{\rho}_{1 s}^{2}\right)\right] \\
& \times C_{l m}\left(B, \rho_{1 s}, \rho_{2 s}, r_{1}, r_{2}\right) \exp \left[j l\left(\theta_{2}-\theta_{1}\right)\right]
\end{aligned}
$$

with

$$
a=\frac{\partial^{2}}{\partial r_{1}^{2}} f\left(\rho_{1}, \rho_{2}\right)=-\frac{A^{*}}{B^{*}}, b=\frac{\partial^{2}}{\partial r_{2}^{2}} f\left(\rho_{1}, \rho_{2}\right)=\frac{A}{B}, \rho_{1 s}=\frac{\beta B^{*}}{A^{*}}, \quad \rho_{2 s}=\frac{\beta B}{A} .
$$

We call the stationary phase points for $\rho_{1 s}$ and $\rho_{2 s}$ are roots of equation:

$$
\frac{\partial}{\partial \rho_{1}} f\left(\rho_{1}, \rho_{2}\right)=\frac{\partial}{\partial \rho_{2}} f\left(\rho_{1}, \rho_{2}\right)=0 .
$$

The transfer matrix for a MGSM beam passing through an axicon is given as [36]:

$$
\left(\begin{array}{ll}
A & B \\
C & D
\end{array}\right)=\left(\begin{array}{ll}
1 & z \\
0 & 1
\end{array}\right) .
$$

Replacing the elements of the transfer matrix in Equation (11) with the elements in Equation (14), we can obtain the final analytical expression for the average intensity of a MGSM beam focused by an axicon:

$$
\begin{aligned}
I(\mathbf{r}, z)= & \frac{2 \pi k}{z^{2} \sqrt{|a b| C_{0}}} \rho_{1 s} \rho_{2 s} \exp \left[j k f\left(\rho_{1 s}, \rho_{2 s}\right)\right] \\
& \times \sum_{m=1}^{M} \sum_{l=-\infty}^{\infty}\left(\begin{array}{c}
M \\
m
\end{array}\right) \frac{(-1)^{m-1}}{m} \exp \left[-\frac{1}{2 \Delta_{m}^{2}}\left(\rho_{2 s}^{2}+\rho_{1 s}^{2}\right)\right] \\
& \times C_{l m}\left(z, \rho_{1 s}, \rho_{2 s}, r, r\right) .
\end{aligned}
$$

\section{Numerical Simulation}

In this section, we will analyze the focused intensity of a MGSM beam shaped by an axicon based on Equation (15). The beam parameter $\lambda$, the refractive index $n_{d}$, and the apex angle $\alpha$ of the axicon are set as $\lambda=0.633 \mu \mathrm{m}, \sigma=2.5 \mu \mathrm{m}, n_{d}=1.51630$ and $\alpha=170^{\circ}$.

In order to clarify the axicon's effect on a MGSM beam, as seen in Figures 3 and 4, we first calculated the normalized intensity distributions of a completely coherent Gaussian beam with $\delta=\infty, M=1$ and a partially coherent Gaussian Schell-model (GSM) beam with $\delta=2 \sigma, M=1$. One can see in Figure 3 that the focused field of a completely coherent Gaussian beam displays an optical needle and the transverse intensity profile remains unchanged and demonstrates a non-diffraction property. Figure 4 shows that an optical needle is also formed as the coherence length of the incident beam decreases while the transverse profile of the intensity at different propagation distances varies due to beam diffraction. To give a quantitative evaluation to the effect of the coherence length on the 
diffraction of focused beams, we calculate the full width at half maximum (FWHM) of the intensity along $\mathrm{z}$ for different values of $\delta$, as shown in Figure 5. One sees that the FWHM along the $\mathrm{z}$ axis remains unchanged for a fully coherent beam, which is consistent with the transverse profiles of the intensity shown in Figure 3. For $\delta=2 \sigma$, the FWHM increases slightly with the increase of $z$, as shown in Figure 5. For the smaller value of $\delta$, the focused field of a GSM beam shaped by an axicon diffracts more quickly. As a result, the length of coherence should not be too small to keep the non-diffraction property.
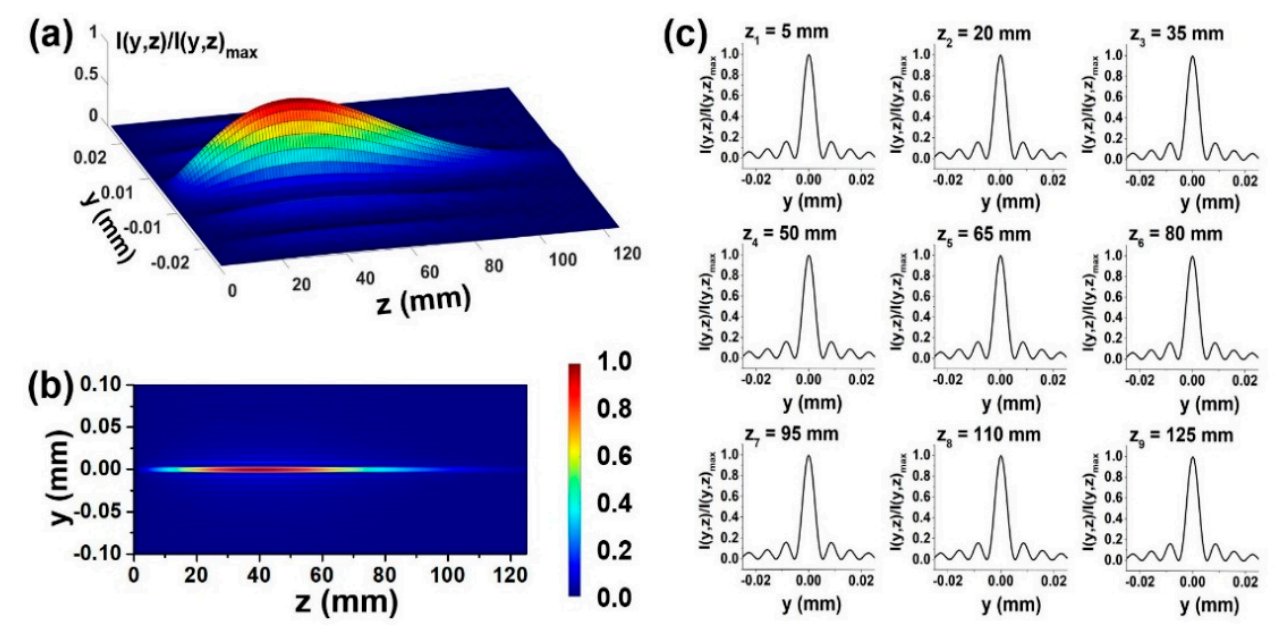

Figure 3. Normalized intensity distribution of a focused fully coherent Gaussian beam (GSM) shaped by an axicon at different propagation distances z: (a) 3-D shaded surface plot of the normalized intensity distribution (b) 2-D density plot of the normalized intensity distribution (c) Cross-section of the normalized intensity distribution.
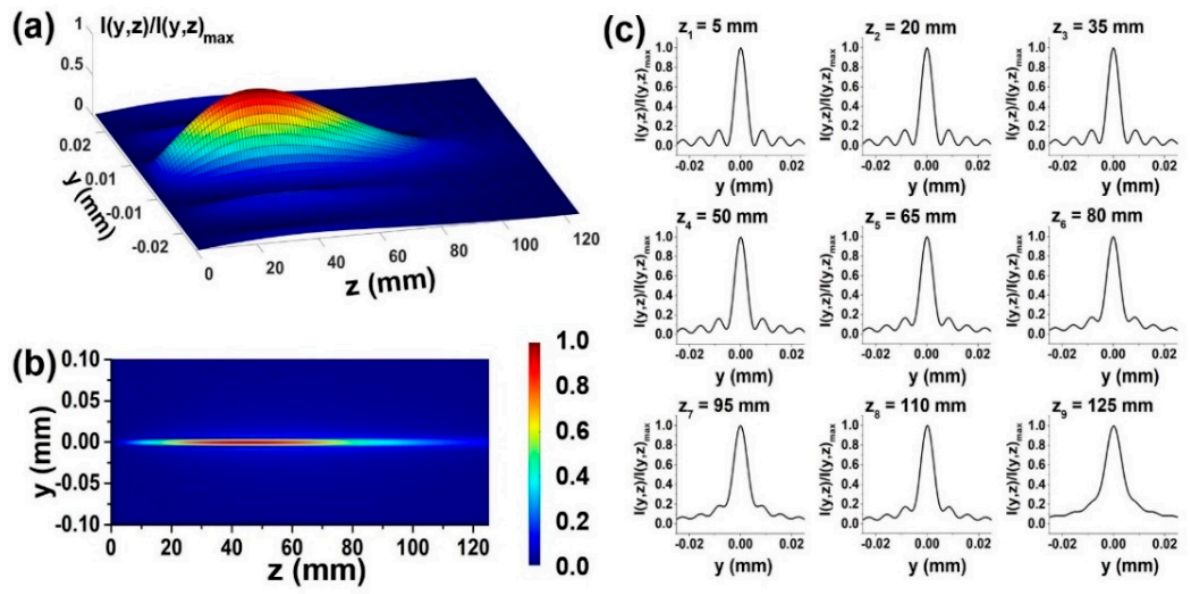

Figure 4. Normalized intensity distribution of a focused partially coherent Gaussian Schell-model beam shaped by an axicon at different propagation distances $\mathrm{z}$ with $\delta=2 \sigma$ : (a) 3-D shaded surface plot of the normalized intensity distribution (b) 2-D density plot of the normalized intensity distribution (c) Cross-section of the normalized intensity distribution. 


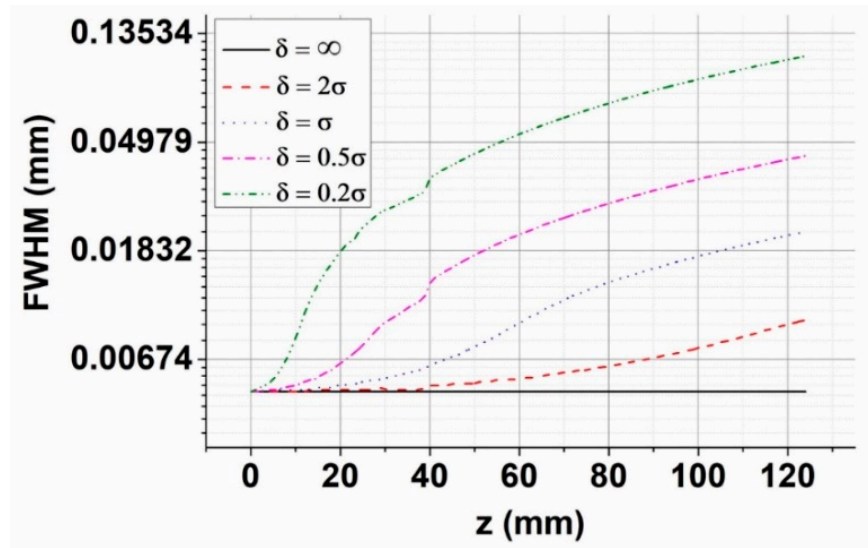

Figure 5. Full width at half maximum (FWHM) of a focused GSM beam shaped by an axicon at different propagation distances $\mathrm{z}$ for different $\delta$.

Next, we concentrated our attention on the focused field of a MGSM beam passing through an axicon. Figure 6 shows the normalized intensity generated by a MGSM beam with $M=4$ and $\delta=0.2 \sigma$. One can see that the focused field of a MGSM beam diffracts more rapidly than that of a GSM beam. The flat-topped profile emerges at $\mathrm{z}=18 \mathrm{~mm}$ and from $\mathrm{z}=18 \mathrm{~mm}$ to $\mathrm{z}=22 \mathrm{~mm}$ the beam profile remains flat-topped. The axicon lens plays the role of extending the longitudinal range of the flat-topped spatial profile. As we increase the beam order $M$ to 12 in Figure 7, the focused field experiences a more complicated variance. The spatial profile varies from a concave top at $\mathrm{z}=14 \mathrm{~mm}$ to a flat-top at $\mathrm{z}=18 \mathrm{~mm}$ and then to a cusp top at $\mathrm{z}=20 \mathrm{~mm}$. It can be concluded that the focused field of a partially coherent beam is closely related to the characteristic parameters of a correlation function. The FWHM of different beam orders $M$ with $\delta=0.2 \sigma$ in Figure 8 clearly demonstrates that as the beam order increases, the focused field diffracts more rapidly.

(a)
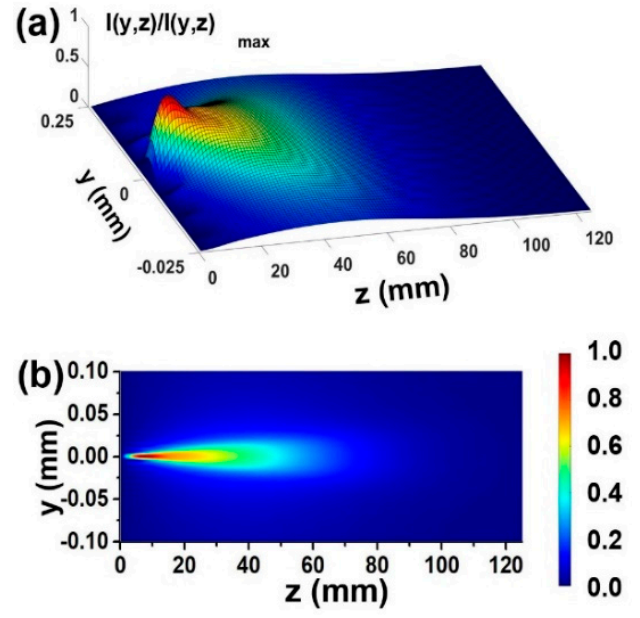

(c)
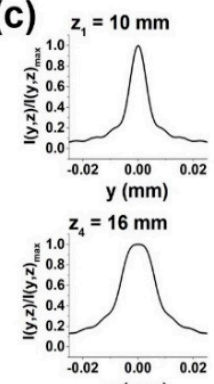

$y(\mathrm{~mm})$

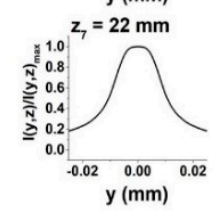

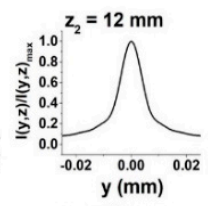
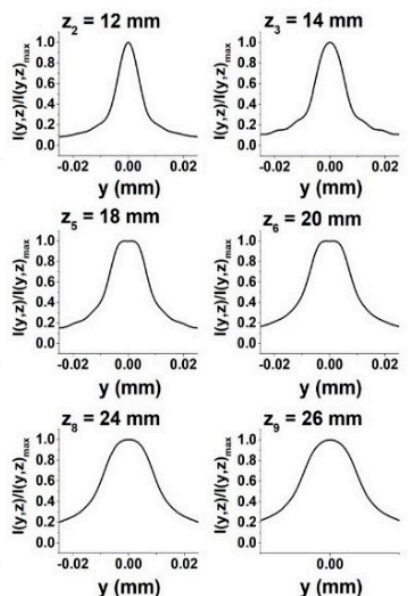

Figure 6. Normalized intensity distribution of a focused MGSM beam shaped by an axicon at different propagation distances z with $M=4, \delta=0.2 \sigma$ : (a) 3-D shaded surface plot of normalized intensity distribution (b) 2-D density plots of normalized intensity distribution (c) Cross-sections of normalized intensity distribution. 
(a) ${ }^{1}$
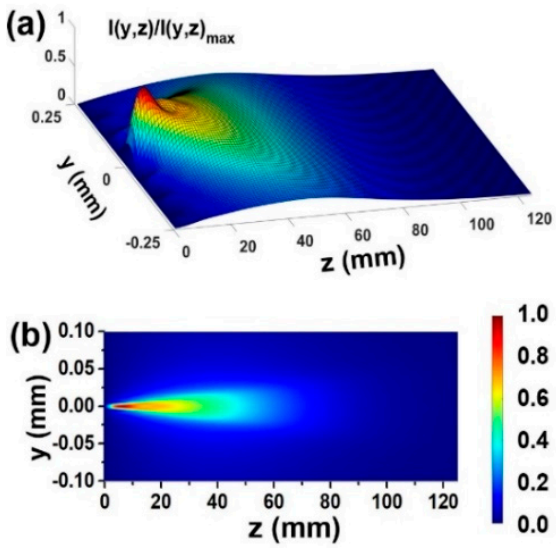

(c)

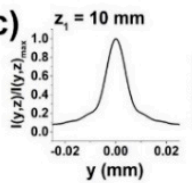

$0.02 \quad 0.00$
$y(m m)$
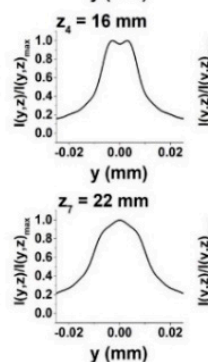
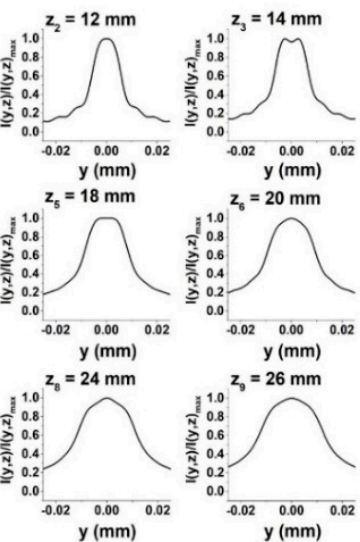

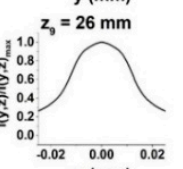

Figure 7. Normalized intensity distribution of a focused MGSM beam shaped by an axicon at different propagation distances z with $M=12, \delta=0.2 \sigma$ : (a) 3-D shaded surface plot of normalized intensity distribution (b) 2-D density plots of normalized intensity distribution (c) Cross-sections of normalized intensity distribution.

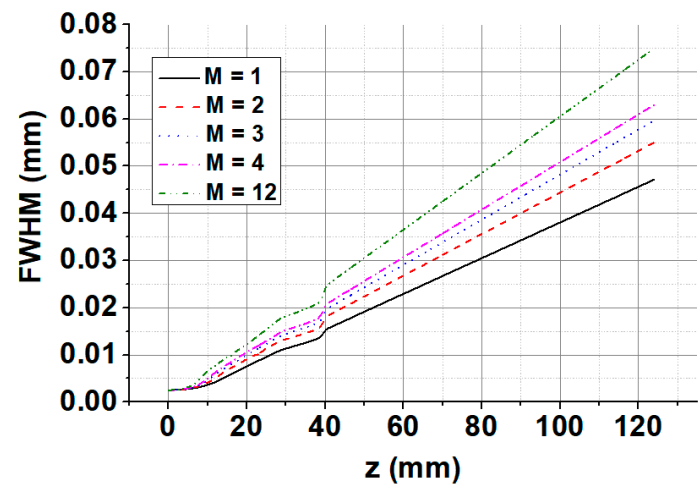

Figure 8. Full width at half maximum (FWHM) of a focused MGSM beam shaped by an axicon at different propagation distances $\mathrm{z}$ with $\delta=0.2 \sigma$ for different beam orders $\mathrm{M}$.

For a non-conventional partially coherent beam, the structure of a correlation function also induces the effect of focal shift [39]. The relative focal shift is defined as:

$$
z_{f}=-\frac{z_{\max }-z_{G \max }}{z_{G \max }}
$$

where $z_{\max }$ and $z_{G \max }$ are the intensity maxima position of a MGSM beam and the corresponding coherent Gaussian light focused by an axicon respectively. It is obvious from Figure 9 that either the lower value of coherence or the larger beam order leads to the increment of relative focal shift.

From above simulation results, we can generate an optical needle with a flat-topped spatial profile and long focal depth by focusing a MGSM beam with an axicon. The advantages of the formed optical needle are that the focal shift and focal depth can be modulated by varying the width of the degree of coherence (DOC) and the characteristic parameters of a correlation function. 

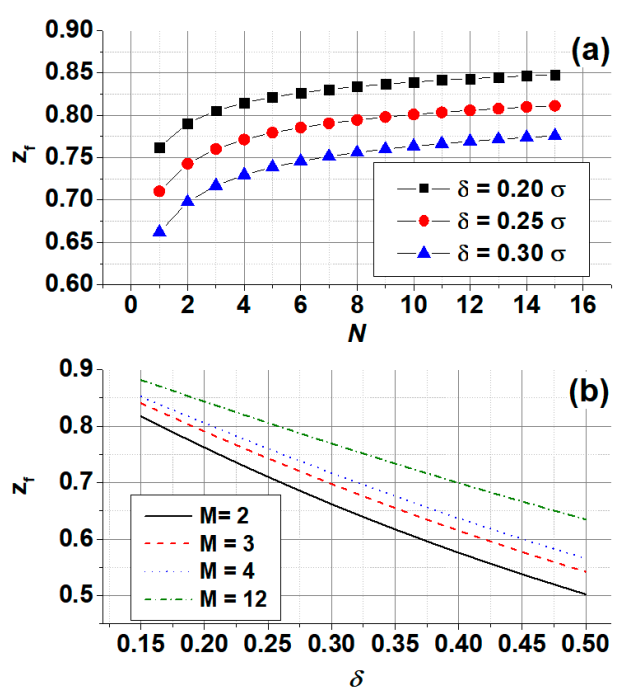

Figure 9. Relative focal shift of a focused MGSM beam shaped by an axicon: (a) for different $\delta$ (b) for different beam orders $\mathrm{M}$.

\section{Conclusions}

Appling the generalized Huygens-Fresnel principle and the stationary phase method, the CSD of a nonconventional partially coherent beam called a MGSM beam passing through an axicon lens was derived in detail. The simulation result, based on the obtained equation, showed that an optical needle with a flat-topped spatial profile and long focal depth can be generated. Compared with the complete coherent light and conventional partially coherent light illumination, a MGSM beam illumination has the advantage not only in the prescribed spatial intensity profile, but also in the convenient control of the focal shift and focal depth. Our results will be useful for electron acceleration [40], particle trapping [41], fiber coupling and percussion drilling [42], where a partially coherent flat-topped beam spot is required.

Author Contributions: Data curation, M.Z., L.G.; writing (original draft), M.Z., L.G.; writing, M.Z., L.G., L.L., Y.C.; supervision, L.L., Y.C.; project administration, L.L., Y.C. All authors read and approved the final manuscript.

Funding: This work was supported by the National Natural Science Foundation of China (91750201, 11525418, 11774251, 11804198), the Key Lab Foundation of The Modern Optical Technology of Jiangsu Province, Soochow University, under Grant No. KJS1606, Characteristic Innovation Project (Natural Science) of the Education Department of Guangdong Province (2017KTSCX114).

Conflicts of Interest: The authors declare no conflict of interest.

\section{References}

1. McLeod, J.H. The Axicon: A new type of optical element. J. Opt. Soc. Am. A 1954, 44, 592-597. [CrossRef]

2. Rioux, M.; Tremblay, R.; Belange, P.A. Linear, annular, and radial focusing with axicons and applications to laser machining. Appl. Opt. 1978, 17, 1532-1536. [CrossRef]

3. Belange, P.A.; Rioux, M. Ring pattern of a lens-axicon doublet illuminated by a Gaussian beam. Appl. Opt. 1978, 17, 1080-1086. [CrossRef]

4. McLeod, J.H. Axicons and Their Uses. J. Opt. Soc. Am. A 1960, 50, 166-169. [CrossRef]

5. Arimoto, R.; Saloma, C.; Tanaka, T.; Kawata, S. Imaging properties of axicon in a scanning optical system. Appl. Opt. 1992, 17, 6653-6657. [CrossRef]

6. Durnin, J. Exact solutions for nondiffracting beams. I. The scalar theory. J. Opt. Soc. Am. A 1987, 4, 651-654. [CrossRef]

7. Durnin, J.; Miceli, J.J; Eberly, H.J. Production and uses of diffractionless beams. Phys. Rev. Lett. 1987, 58, 1499-1501. [CrossRef]

8. Durnin, J.; Miceli, J.J.; Eberly, H.J. Durnin, Miceli, and Eberly reply. Phys. Rev. Lett. 1991, 66, 838. [CrossRef] [PubMed] 
9. Herman, M.R.; Wiggins, A.T. Production and uses of diffractionless beams. J. Opt. Soc. Am. A 1991, 8 , 932-942. [CrossRef]

10. Popov, S.Y.; Friberg, A.T. Design of diffractive axicons for partially coherent light. Opt. Lett. 1998, 23, 1639-1641. [CrossRef] [PubMed]

11. Friberg, A.T.; Popov, S.Y. Effects of partial spatial coherence with uniform-intensity diffractive axicons. J. Opt. Soc. Am. A 1999, 16, 1049-1058. [CrossRef]

12. Shukri, M.; Alkelly, A.A.; Alarify, Y.S. Apodized design of diffractive axicons for twisted partially coherent light. Appl. Opt. 2013, 52, 1881-1887. [CrossRef]

13. Alkelly, A.A.; Shukri, M.; Alarify, Y.S. Influences of twist phenomenon of partially coherent field with uniform-intensity diffractive axicons. J. Opt. Soc. Am. A 2012, 29, 417-425. [CrossRef]

14. Pu, J.; Liu, X.; Nemot, S. Partially coherent bottle beams. Opt. Commun. 2005, 252, 7-11. [CrossRef]

15. Pu, J.; Dong, M.; Wang, T. Generation of adjustable partially coherent bottle beams by use of an axicon-lens system. Appl. Opt. 2006, 45, 7553-7556. [CrossRef]

16. Alkelly, A.A.; Shukri, M.; Alarify, Y.S. Intensity distribution and focal depth of axicon illuminated by Gaussian Schell-model beam. Opt. Commun. 2011, 284, 4658-4662. [CrossRef]

17. Cai, Y.; Chen, Y.; Yu, J.; Liu, X.; Liu, L. Generation of Partially Coherent Beams. Prog. Opt. 2017, 62, $157-223$. [CrossRef]

18. Lu, X.; Shao, Y.; Zhao, C.; Konijnenberg, S.; Zhu, X.; Tang, Y.; Cai, Y.; Urbach, H.P. Noniterative spatially partially coherent diffractive imaging using pinhole array mask. Adv. Photonics. 2019, 1, 016005. [CrossRef]

19. Cai, Y.; Chen, Y.Y.; Wang, F. Generation and propagation of partially coherent beams with nonconventional correlation functions: A review [Invited]. J. Opt. Soc. Am. A 2014, 31, 2083-2096. [CrossRef]

20. Wang, F.; Liu, X.; Yuan, Y.; Cai, Y. Experimental generation of partially coherent beams with different complex degrees of coherence. Opt. Lett. 2013, 38, 1814-1816. [CrossRef]

21. Liu, X.; Liu, L.; Wang, F.; Cai, Y. Generation of a flexible far-field anomalous hollow beam spot through superposition of two partially coherent sources with different degrees of coherence. Opt. Commun. 2018, 428, 69-76. [CrossRef]

22. Chen, Y.; Wang, F.; Liu, L.; Zhao, C.; Cai, Y.; Korotkova, O. Generation and propagation of a partially coherent vector beam with special correlation functions. Phys. Rev. A. 2014, 89, 013801. [CrossRef]

23. Liang, C.; Wang, F.; Liu, L.; Cai, Y.; Korotkova, O. Experimental generation of Cosine-Gaussian-correlated Schell-model beams with rectangular symmetry. Opt. Lett. 2014, 39, 769-772. [CrossRef]

24. Chen, Y.; Gu, J.; Wang, F.; Cai, Y. Self-splitting properties of a Hermite-Gaussian correlated Schell-model beam. Phys. Rev. A 2015, 91, 013823. [CrossRef]

25. Chen, Y.; Wang, F.; Yu, J.; Liu, L.; Cai, Y. Vector Hermite-Gaussian correlated Schell-model beam. Opt. Express 2016, 24, 15232-15250. [CrossRef]

26. Yu, J.; Cai, Y.; Gbur, G. Rectangular Hermite non-uniformly correlated beams and its propagation properties. Opt. Express 2018, 26, 27894-27906. [CrossRef]

27. Liu, X.; Liu, L.; Peng, X.; Liu, L.; Wang, F.; Gao, Y.; Cai, Y. Partially coherent vortex beam with periodical coherence properties. J. Quant. Spectrosc. Radiat. Transf. 2019, 222, 138-144. [CrossRef]

28. Yu, J.; Wang, F.; Liu, L.; Cai, Y.; Gbur, G. Propagation properties of Hermite non-uniformly correlated beams in turbulence. Opt. Express 2018, 26, 16333-16343. [CrossRef]

29. Peng, X.; Lu, X.; Liu, X.; Zhao, C.; Lin, R.; Liu, L.; Cai, Y. Generation and propagation of a Hermite-Gaussian correlated Schell-model LG0l beam. Appl. Sci. 2019, 9, 610. [CrossRef]

30. Guo, L.; Chen, L.; Lin, R.; Zhang, M.; Gao, Y.; Cai, Y. Generation of an adjustable optical cage through focusing an apertured Bessel-Gaussian correlated Schell-model beam. Appl. Sci. 2019, 9, 550. [CrossRef]

31. Sahin, S.; Korotkova, O. Light sources generating far fields with tunable flat profiles. Opt. Lett. 2012, 37, 2970-2972. [CrossRef]

32. Korotkova, O. Random sources for rectangular far fields. Opt. Lett. 2014, 39, 64-67. [CrossRef]

33. Korotkova, O. Can a sphere scatter light producing rectangular intensity patterns? Opt. Lett. 2015, 40, 1709-1712. [CrossRef]

34. Zhang, Y.; Cai, Y. Random source generating far field with elliptical flat-topped beam profile. J. Opt. 2014, 16, 075704. [CrossRef]

35. Wang, F.; Liang, C.; Cai, Y. Generalized multi-Gaussian correlated Schell-model beam: From theory to experiment. Opt. Express 2014, 22, 23456-23464. [CrossRef] 
36. Collins, S.A. Lens-system diffraction integral written in terms of matrix optics. J. Opt. Soc. Am. A 1970, 60, 1168-1177. [CrossRef]

37. Lin, Q.; Cai, Y. Tensor ABCD law for partially coherent twisted anisotropic Gaussian-Schell model beams. Opt. Lett. 2002, 27, 216-218. [CrossRef]

38. Friberg, A.T. Stationary-phase analysis of generalized axicons. J. Opt. Soc. Am. A 1996, 13, 743-750. [CrossRef]

39. Zhang, M.H.; Chen, Y.; Cai, Y.; Liu, L. Effect of the correlation function on the focal shift of a partially coherent beam. J. Opt. Soc. Am. A 2016, 33, 2509-2515. [CrossRef]

40. Wang, W.; Wang, X.P.; Ho, Y.K.; Kong, Q.; Chen, Z.; Gu, Y.; Wang, S.J. Field description and electron acceleration of focused flattened Gaussian laser beams. Europhys. Lett. 2006, 73, 211-217. [CrossRef]

41. Zhao, C.; Cai, Y.; Lu, X.; Eyyuboğlu, H.T. Radiation force of coherent and partially coherent flat-topped beams on a Rayleigh particle. Opt. Express 2009, 17, 1753-1765. [CrossRef]

42. Coutts, D.W. Double-pass copper vapor laser master-oscillator power-amplifier systems: Generation of flat-top focused beams for fiber coupling and percussion drilling. IEEE J. Quantum Electron. 2002, 38, 1217-1224. [CrossRef]

(C) 2019 by the authors. Licensee MDPI, Basel, Switzerland. This article is an open access article distributed under the terms and conditions of the Creative Commons Attribution (CC BY) license (http://creativecommons.org/licenses/by/4.0/). 